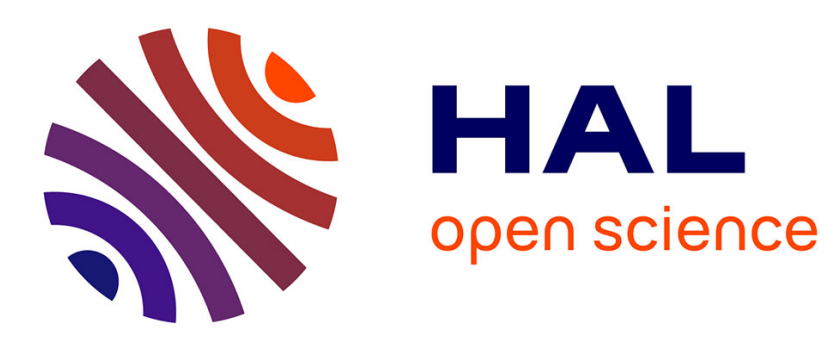

\title{
Fractals et structure des réseaux urbains d'assainissement eau pluviale
}

\author{
Serge Michel Jacques Thibault
}

\section{To cite this version:}

Serge Michel Jacques Thibault. Fractals et structure des réseaux urbains d'assainissement eau pluviale. Flux - Cahiers scientifiques internationaux Réseaux et territoires, 1991, 4, pp.5-14. hal-00654988

\section{HAL Id: hal-00654988 \\ https://hal.science/hal-00654988}

Submitted on 24 Dec 2011

HAL is a multi-disciplinary open access archive for the deposit and dissemination of scientific research documents, whether they are published or not. The documents may come from teaching and research institutions in France or abroad, or from public or private research centers.
L'archive ouverte pluridisciplinaire HAL, est destinée au dépôt et à la diffusion de documents scientifiques de niveau recherche, publiés ou non, émanant des établissements d'enseignement et de recherche français ou étrangers, des laboratoires publics ou privés. 


\title{
Fractals et structure des réseaux urbains d'assainissement eau
}

\section{pluviale}

\author{
In: Flux n4, 1991. pp. 5-14.
}

\begin{abstract}
Serge THIBAULT, Fractals and the structure of urban rainwater run-off systems. The evolution of the concept and management of rainwater run-off systems is the starting point for this research, which consists in linking the hydrological function of urban drainage pools to the morphology of the drainage systems. This latter element can be formulated as a model by means of fractal dimension theory. Thus, a system is defined as an arrangement of sections composing a spatial form, whose dimension can be understood as between one and two. This model, while taking into account the greater or lesser complexity of the object in question, is at the same time characterized by properties which illustrate an urban form via a particular technical network. We hope that this approach can be extended to other technical networks.
\end{abstract}

\section{Résumé}

Serge THIBAULT, Fractals et structure des réseaux urbains d'assainissement eau pluviale. L'évolution de la conception et de la gestion des réseaux d'assainissement eau pluviale est à l'origine d'un travail de recherche qui a consisté à relier le fonctionnement hydrologique des bassins versants urbains à la morphologie des réseaux de drainage. Celle-ci peut être modélisée à l'aide de la théorie de la dimension fractale. Ainsi un réseau peut être défini comme un agencement de tronçons formant une forme spatiale de dimension comprise entre un et deux. Ce modèle, tout en rendant compte de la plus ou moins grande complexité de l'objet, possède des propriétés qui illustrent une forme urbaine via un réseau technique particulier. Nous souhaitons que cette approche puisse être étendue à d'autres réseaux techniques.

Citer ce document / Cite this document :

Thibault Serge. Fractals et structure des réseaux urbains d'assainissement eau pluviale. In: Flux n4, 1991. pp. 5-14.

doi : 10.3406/flux.1991.1149

http://www.persee.fr/web/revues/home/prescript/article/flux_1154-2721_1991_num_7_4_1149 


\title{
FRACTALS ET STRUCTURE DES RÉSEAUX URBAINS
}

\section{D'ASSAINISSEMENT EAU PLUVIALE}

\author{
SERGE THIBAULT
}

Serge THIBAULT est Docteur d'Etat en Sciences Pbysiques spécialité Génie $U r$ bain. Professeur au Centre d'Etudes Supérieures d'Aménagement (CESA) de l'Université de Tours, son enseignement et ses recherches portent sur le génie urbain et le développement des sciences de la conception en Aménagement et Urbanisme.

Le milieu urbain peut être conçu à partir de la notion d'éco-système, l'urbatope étant en partie constitué de réseaux techniques. Nous pouvons dire que l'ensemble des réseaux techniques participe de l'autonomisation du milieu urbain vis-à-vis de son environnement, chacun d'eux ayant une finalité spécifique dans cette recherche d'autonomie.

Ainsi les réseaux d'assainissement pluviaux ont été conçus pour assurer le fonctionnement du milieu urbain lorsque celui-ci est soumis aux flux d'eau d'origine météorique, l'évacuation de ces eaux n'étant plus assurée de par la disparition du réseau hydrographique naturel, conséquence de l'imperméabilisation des sols. L'accroissement du phénomène d'agglomération et du développement des activités en milieu urbain à partir des années 1960 a introduit un basculement dans la conception et la gestion des réseaux d'assainissement.

En effet, jusqu'à cette époque et de par les choix techniques retenus vers la fin du dix-neuvième siècle (principe du "tout à l'égout" pour une évacuation systématique), l'urbatope se constitue en intégrant systématiquement ce réseau d'évacuation des eaux pluviales (et usées). La conception s'identifie alors uniquement à un choix de trajet et à un dimensionnement des canalisations à l'aide d'une formule "réglementaire" dite formule de Caquot qui permet d'estimer la valeur du débit de pointe à évacuer par le réseau".

Les possibilités de développement dues à l'autonomisation du milicu urbain, vont avoir un effet "pervers" sur les réseaux d'assainissement de certaines agglomérations. En effet ceux-ci vont se révéler insuffisants pour drainer les zones nouvelles.

Pour faire face à cette contradiction, nous pouvons dire que les concepteurs et gestionnaires, tout en revenant sur 


\section{FLUX 4 Avril-Juin 1991}

le principe d'évacuation systématique des eaux pluviales, vont commencer à concevoir l'évolution des réseaux d'assainissement à partir d'un principe de régulation :

$$
\begin{array}{lll}
\text { évolution } & \rightarrow & \text { évolution } \\
\text { de l'urbain } & \leftarrow & \text { du réseau }
\end{array}
$$

et non plus à partir du principe de cause à effet du type :

$$
\begin{aligned}
& \begin{array}{l}
\text { évolution } \\
\text { de l'urbain }
\end{array} \\
& \rightarrow \quad \begin{array}{l}
\text { développement } \\
\text { du réseau }
\end{array}
\end{aligned}
$$

Pour ce faire, la science de l'hydrologie urbaine va développer des modèles permettant de représenter de façon nouvelle, par rapport à la formule de Caquot, le comportement hydrologique des réseaux d'assainissement. C'est initialement dans ce cadre que nous avons bâti un modèle de transformation pluie-débit basé sur la modélisation de la structure des réseaux d'assainissement. Nous appelons structure l'agencement des tronçons de canalisations formant réseau.

Dans ce texte, nous nous en tiendrons à la présentation de ce modèle de structure, renvoyant le lecteur intéressé par des aspects hydrologiques à notre thèse ${ }^{2}$.

\section{PRINCIPE DE MODELISATION}

Que ce soit pour la simulation du fonctionnement hydraulique d'un réseau ou pour la conception d'un réseau d'évacuation, les modèles de transformation pluie-débit ne sont pas basés sur la dualité

$$
\text { structure } \leftrightarrow \text { fonction }
$$

où le transformateur, c'est-à-dire le bassin versant drainé, serait appréhendé comme une boîte noire:

$$
\begin{aligned}
& \underset{\substack{\text { intensité } \\
\text { pluvieuse } \\
I(t)}}{ } \rightarrow \text { débit } \\
& Q(t)
\end{aligned}
$$

De ce fait, la modélisation de cette transformation est basée sur le schéma suivant ${ }^{3}$ :

* Recherche de l'opération $O$ représentant au mieux la relation entre $Q(t)$ et $I(t)$

- Calage des paramètres de l'opérateur à partir de certaines grandeurs physiques mesurables (surface, imperméabilisation, etc.)

Pour dépasser les limites de l'approche purement expérimentale, nous avons élaboré une théorie basée sur la relation modélisation de la fonc. tion/modélisation de la structure, la structure des réseaux d'assainissement étant un mélange de structures ramifiées et de structures maillées.

Ces réseaux sont un ensemble de canalisations (tronçons) reliées entre elles par des regards (cheminées en langue lyonnaise). Vis-à-vis d'une approche hydrodynamique, les noeuds du réseau sont les lieux de discontinuités hydrauliques.

\section{MODELE FRACTAL}

La nécessité de simuler le fonctionnement hydrologique de bassins sans détailler le fonctionnement hydraulique du réseau interne tronçon par tronçon, les réflexions qui se développent sur l'interaction entre l'évolution du milieu urbain et des réseaux techniques révèlent l'importance de la création de modèles permettant de concevoir ce qu'est la morphologie d'un réseau telle que celle d'un réseau d'assainissement.

En considérant qu'un tronçon est une figure de dimension euclidienne un, la question posée est la suivante :

"Est-ce qu'une organisation de segments de dimensicn un peut être représentée par un objet géométrique qui rende compte de la complexité structurelle d'un réseau?",

celui-ci étant une figure orientée par le sens des écoulements, des tronçons terminaux vers le point exutoire considéré. 


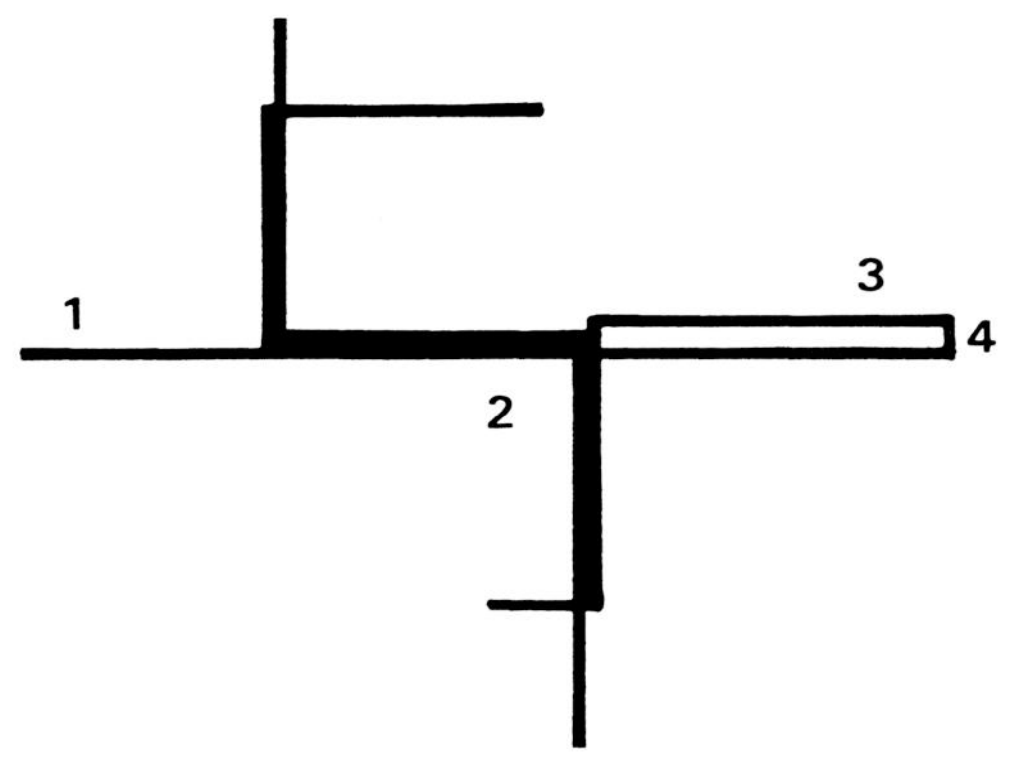

FIGURE 1: ORDONNANCEMENT DE STRAHLER

Pour aborder cette modélisation, nous nous sommes orienté vers l'usage des concepts développés par la théorie de la dimension fractale, puisque les modèles notamment développées par Horton et Strahler aux U.S.A. pour représenter la structure des réseaux hydrographiques ramifiés naturels ne rendent pas compte de la complexité de l'objet étudié ${ }^{4}$. Rappelons que ces modèles sont basés sur un ordonnancement des tronçons d'un réseau à partir d'un point aval et d'un décompte du nombre de tronçons par ordres, et qu'ils supposent une progression géométrique des nombres de tronçon par ordre (Figure 1).

Ne rendant pas compte de la structure des réseaux d'assainissement, nous avons créé un processus basé sur la notion de dimension d'homothétie puis d'homothétie statistique permettant de générer des formes voisines de celles observées ${ }^{6}$.

\section{ESPACE DE MINKOWSKI}

L'espace où vont se déployer ces formes n'est pas le plan euclidien mais peut être représenté par les noeuds d'un maillage qui n'est pas forcément régulier.
Ainsi la distance entre deux points quelconques est la longueur du plus petit chemin constitué d'un ensemble de segments reliant ces deux points.

Une boule de centre $A$ et de rayon $R$ contient des noeuds dont la distance au centre est inférieure ou égale à $R$.

La géométrie de cet espace a été développée par Minkowski et est généralement intitulée géométrie du Taxi par les anglo-saxons ${ }^{7}$ (Figure 2).

\section{DIMENSION D'UN RESEAU}

Pour pouvoir appréhender la structure des réseaux d'assainissement, nous avons d'abord cherché à créer des images artificielles. La création d'une image artificielle d'un réseau est basée sur la ramification et l'enchaînement de segments à partir d'un segment initial de longueur $R_{M}$, le processus de création étant un schéma itératif d'interpolations voire d'extrapolations.

Nous considérons que cette longueur $R_{M}$ est également le rayon de la boule au sens de la géométrie précédemment définie, contenant tout le réseau. 


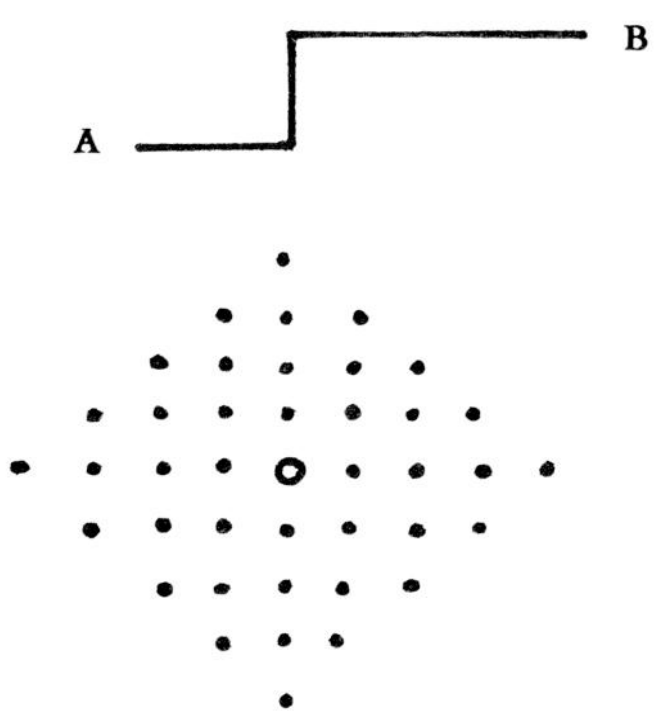

FIGURE 2: ESPACE DE MINKOWSKI
Pour chacun des segments obtenus ce processus est réitéré, chaque phase de réitération étant une interpolation (Figure 3).

Ce processus peut être caractérisé par la dimension d'homothétie suivante ${ }^{8}$ :

$$
D=\log (N) / \log (1 / h)
$$

En considérant maintenant que chacune des parties peut avoir une longueur différente mais que le rapport d'homothétie moyen et le nombre de parties $\mathrm{N}$ créé sont tels que la dimension varie très peu par étape d'interpolation, on engendre des formes dont la structure est cette fois-ci beaucoup plus proche de celles des réseaux observés (Figure 4).

Dans cette boule, centrée sur l'une des extrémités du segment initial, se déploic le réseau.

Pour un rapport d'homothétie donné (h), le seg. ment initial est divisé en $1 / \mathrm{h}$ parties égales. Il possède alors $(1 / \mathrm{h}-1)$ points de ramifications possibles sur lesquels sont ajoutés $N-1 / \mathrm{h}$ parties de longueur $\mathrm{R}_{\mathrm{M}} \mathrm{h}, \mathrm{N}$ étant le nombre de parties à la fin de l'itération.
A une étape donnée du parcours d'interpolation, la forme possède $\mathrm{N}$ segments de longueur moy. enne $1=R_{M} \cdot h$ avec $h$ rapport d'homothétie final. La longueur totale du réseau $L$ est alors égale à: $L=R^{1-D} \cdot R_{M}{ }^{D}$.

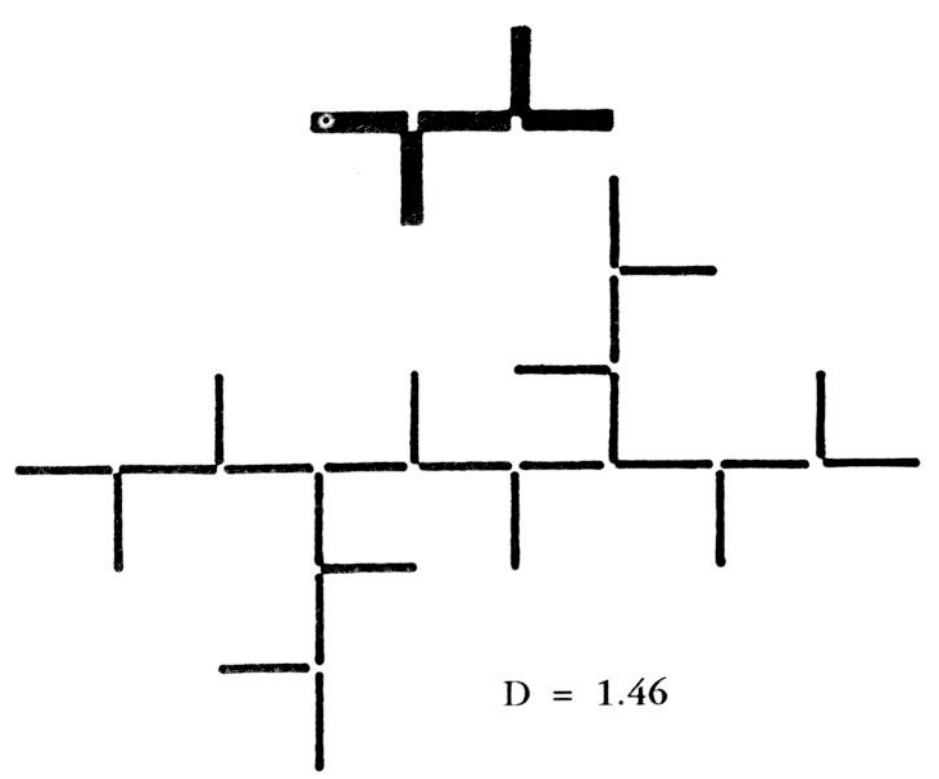




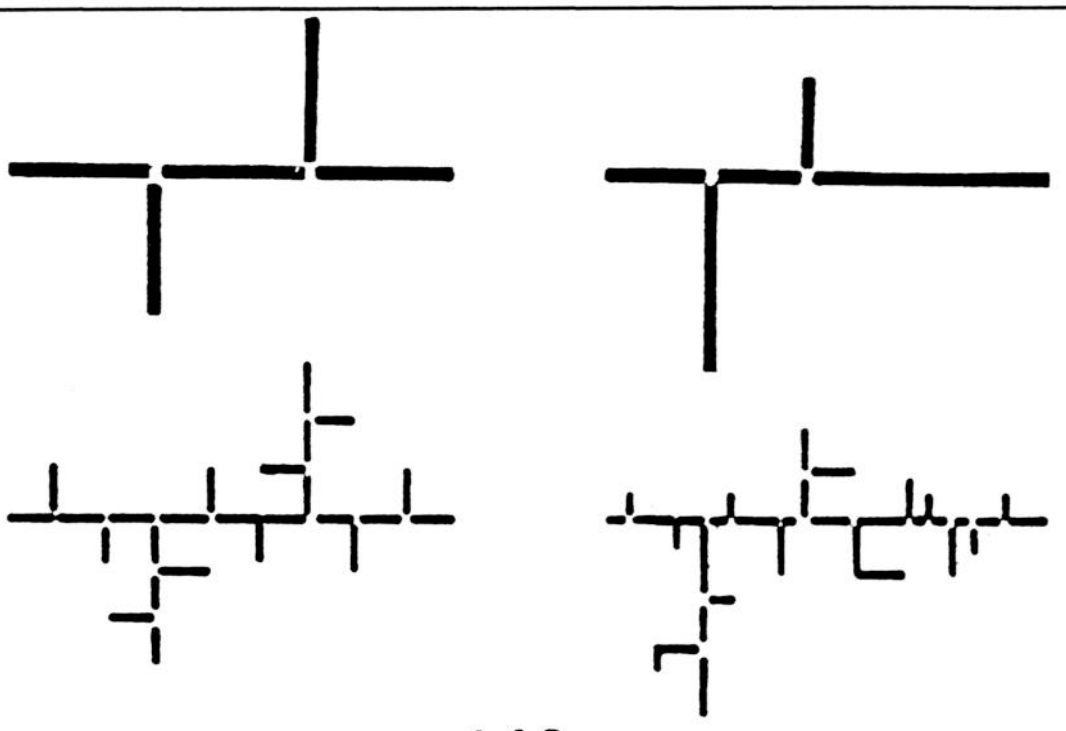

1.46

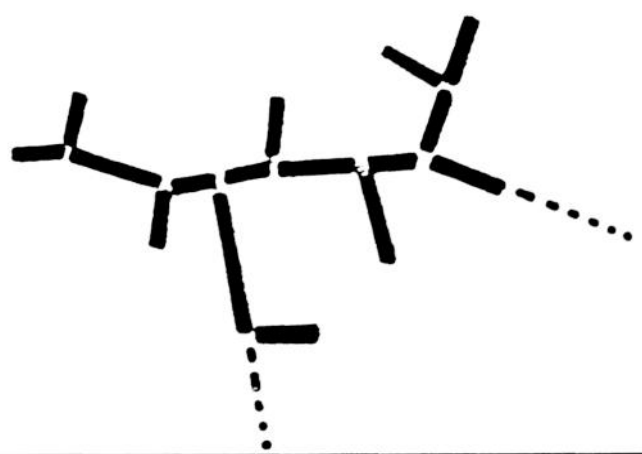

FIGURE 4

Nous avons démontré que la relation $L(R)=1^{1-D}$ $R^{D}$ était une très bonne approximation de la longueur totale de la partie du réseau contenue dans la boule centrée sur l'extrémité du segment initial et de rayon $R, R$ variant de 0 à $R_{M}$. 9

Ce résultat rejoint une propriété des fractals très utilisée pour définir la dimension d'un objet réel ${ }^{10}$.

Maintenant, si $\mathrm{m}$ formes statistiquement identiques vis-à-vis du processus d'interpolation sont mises en parallèle au centre de la boule et sont contenues en elle, nous aurons :

$$
L(R)=m \cdot 1^{1-D} \cdot R^{D}
$$

chacune de ces figures ayant des segments de longueur moyenne 1 (Figure 5).

\section{EVALUATION DE LA DIMENSION D'UN RESEAU}

PRINCIPES

Si la structure des réseaux d'assainissement pouvait être quantifiée par une dimension d'homothétie statistique, ces réseaux s'apparenteraient structurellement à des formes fractales pour lesquelles la représentation serait basée sur une succession d'interpolations en nombre fini, puisque la longueur de la partie ne peut pas être plus petite que la longueur moyenne du tronçon reliant deux regards.

Ces tronçons étant de longueur variable, le modèle structurel est basé sur l'usage d'un processus d'interpolation statistique, qui peut combiner ramifications, enchaînements des parties et mise en parallèle de figures. Ces caractéristiques ne nous 


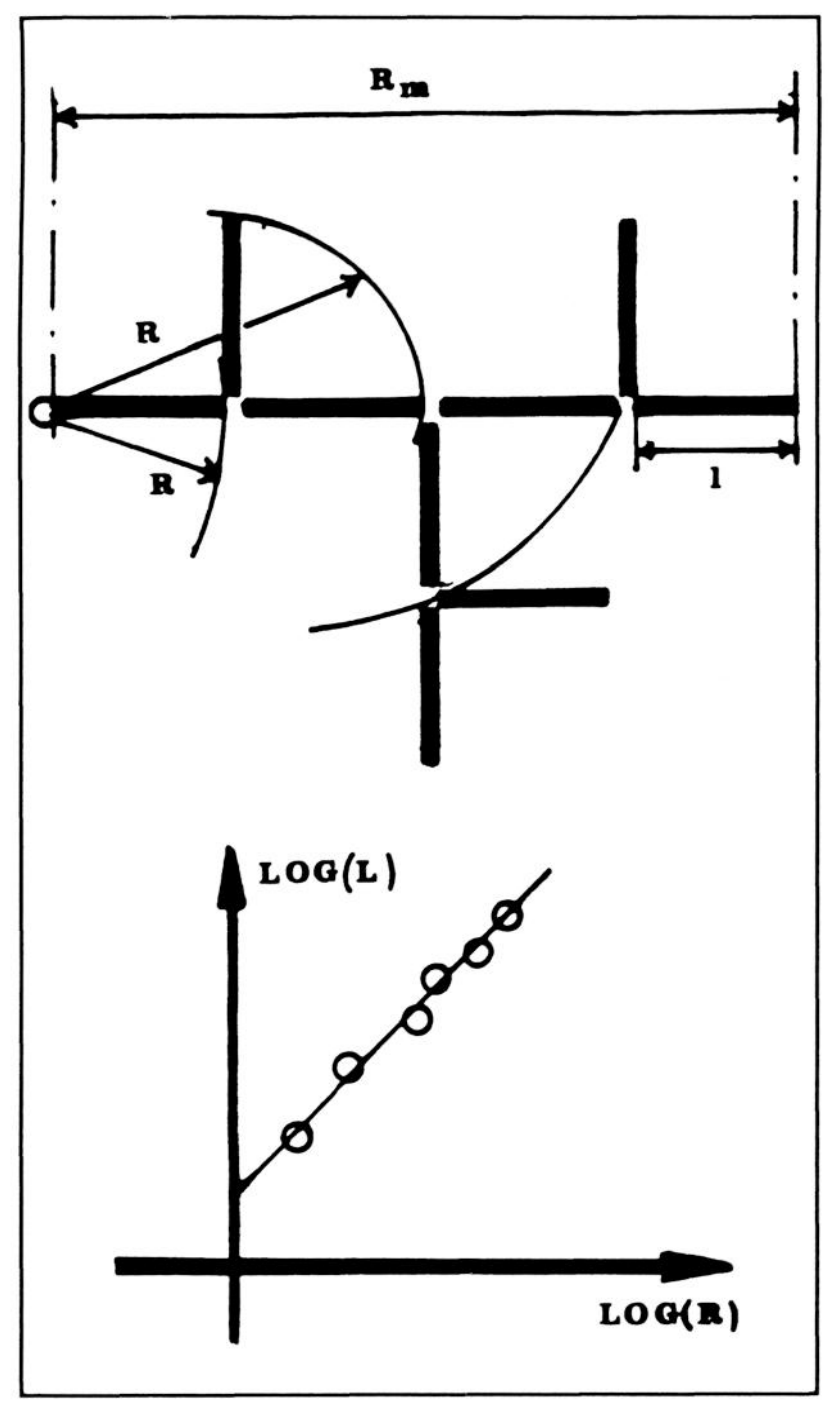

FIGURE 5

permettent pas d'évaluer la possibilité de dimensionner un réseau à partir de la découverte de la structure "élémentaire" qui, interpolée et extrapolée statistiquement, donnerait la structure étudiée.

Si la mesure de l'évolution de la longueur totale du réseau contenu dans une boule de rayon variable, centrée sur un point du réseau considéré comme exutoire peut être correctement approximée par une relation du type :

$$
L(R) \sim a R^{D}
$$

nous pourrons assimiler le réseau à une figure fractale de dimension $\mathrm{D}$ avec $\mathrm{a} \sim \mathrm{m} \mathrm{l}^{1-\mathrm{D}}$ (m nombre de réseaux parallèles à l'exutoire, 1 longueur moyenne du tronçon).
En réalité, ce principe de mesure revient à décomposer tout un réseau, en sous réseaux caractérisés par leur dimension, un exemple de décom. position (ultime ?) étant la décomposition en tronçons élémentaires de dimension unitaire.

La décomposition revient à définir un ensemble de parties pour lesquelles la relation a. $R^{\mathrm{D}}$ est approximativement satisfaite, relation traduisant un principe d'homogénéité structurelle. Comme un réseau d'assainissement urbain peut être décomposé en tronçons élémentaires, le réseau est totalement décomposable, la décomposition n'étant pas unique.

A partir de la représentation informatisée du réseau de la communauté urbaine de Lyon, nous avons pu vérifier que ces principes étaient satisfaits (Fig. 6) et attacher une dimension à tout un ensemble de sous réseaux (Figs. 7-10).

Cette validation est basée sur le calcul d'un écart relatif pondéré par la longueur :

$$
e=\frac{\sum_{i=1}^{m}\left|L_{i}-L_{i}\right|}{\sum_{i=1}^{m} L_{i}}
$$

$\mathrm{L}_{\mathrm{i}}$ : longueur réelle à $\mathrm{Ri} ; \mathrm{L}_{\mathrm{i}}^{*}$ longueur du modèle d'approximation à $\mathrm{Ri}, \mathrm{Ri}$ valeur du rayon de la boule.

Pour le réseau de la commune de Meyzieu, en assimilant le coefficient a de la relation $L(R)=$ $\mathrm{aR}^{\mathrm{D}}$ au produit $\mathrm{ml}^{1-\mathrm{D}}$ avec 1 longueur moyenne des tronçons entre points de ramification $(\sim 180 \mathrm{~m})$, $\mathrm{m}$ vaut 0.62 pour une longueur totale de réseau avoisinant les $50 \mathrm{Km}$.

(On peut interpréter cette valeur comme suit : Cette commune périphérique est caractérisée par 


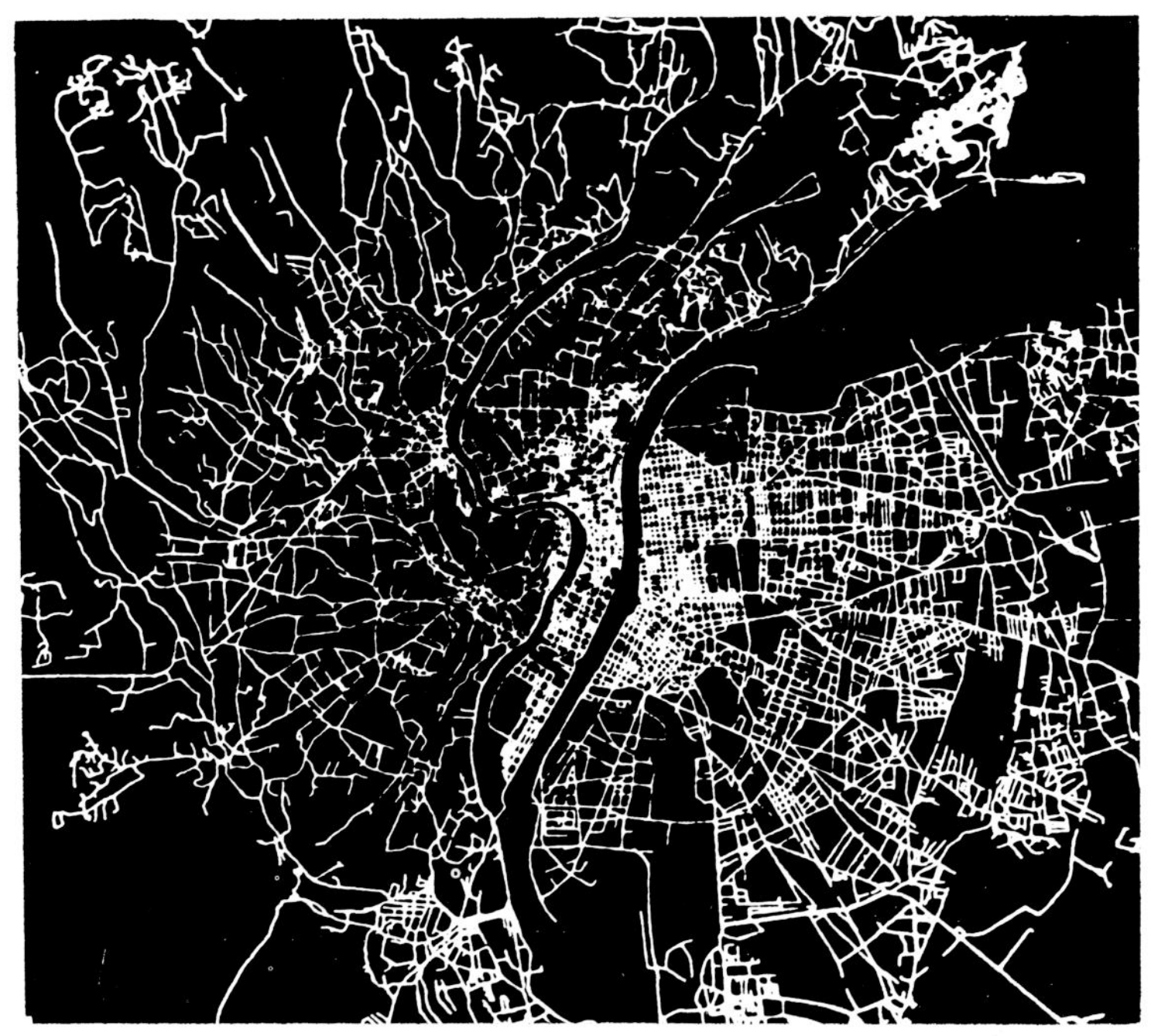

FIGURE 6: IMAGE DU RÉSEAU D'ASSAINISSEMENT DU CENTRE DE LYON

une mixité entre l'urbain et le rural qui se traduit, en terme de modèle, par une interpolation (image du processus d'urbanisation) qui ne serait réaliscé qu'à $62 \%$.)

\section{CONCLUSION}

Même si la structure d'un réseau d'assainissement mélange la ramification et le maillage, le modèle structurel basé sur l'assimilation de ce réseau à une forme fractale permet de représenter très correctement l'objet modélisé en le caractérisant par une dimension comprise entre un et deux, d'autant plus proche de un que le réseau est linéique, d'autant plus proche de deux que celui-ci recouvre la surface considérée. Le type même de réseau de dimension deux est le réseau totalement maillé mis en place sur les zones urbaines à mailles répétitives construites à la fin du 19ème siècle et au début du 20ème (Quartier Brotteaux à Lyon, Plan Cerda pour Barcelone...). Ce modèle illustre la complexité morphologique de l'objet réseau. Rappelons que notre objectif premier, quand nous avons commencé ce travail, était de relier structure et fonc. tionnement. Ceci nous a conduit à retenir la longueur comme variable pertinente pour modéliser le déploiement spatial de l'objet. Si l'on considère l'objet fonctionnant, sa structure devrait être définie par les flux qui le parcourent soit en l'occurrence les vitesses des écoulements liés aux débits 
eaux fluviales. Cette vitesse, en ce qui concerne les réseaux d'assainissement, est une grandeur physique pertinente. Mais l'inconvénient majeur, pour la formalisation de la relation structure-fonction, est qu'il n'y a plus une scule structure mais autant que de flux qui parcourent l'objet, puisque les débits et vitesses associées sont variables. Ie passage de l'impossible au possible que nous avons opéré est dû à la finalité de la modélisation, c'est-à-dire représenter le fonctionnement d'un réseau pour des valeurs de débit pour lesquels la vitesse moyenne varic peu.

Cette démarche spécifie le travail effectué et pose le problème de la transposition pour l'étude de réseaux techniques urbains autres.

S'il s'agit de modéliser la morphologie de l'objet, il faut initialement convenir de la variable pertinente d'une telle description. Ft nous pouvons concevoir que celle-ci est spécifique à chacun des réseaux; la longueur, le temps pour certains, le nombre d'information pour d'autres, etc.

Enfin, si l'on examine les travaux existants sur la modélisation des réseau techniques et en particulier l'ouvrage "Systèmes, réscaux et territoires"11, nous pouvons dire que chacune de ces modélisations est par nature dépendante des propriétés que l'on veut définir sur le réseau support. Un travail de recherche plus transversal devrait être mené sur la morphologic des réscaux pour définir les spécificités et les propriétés communes de ccux-ci.

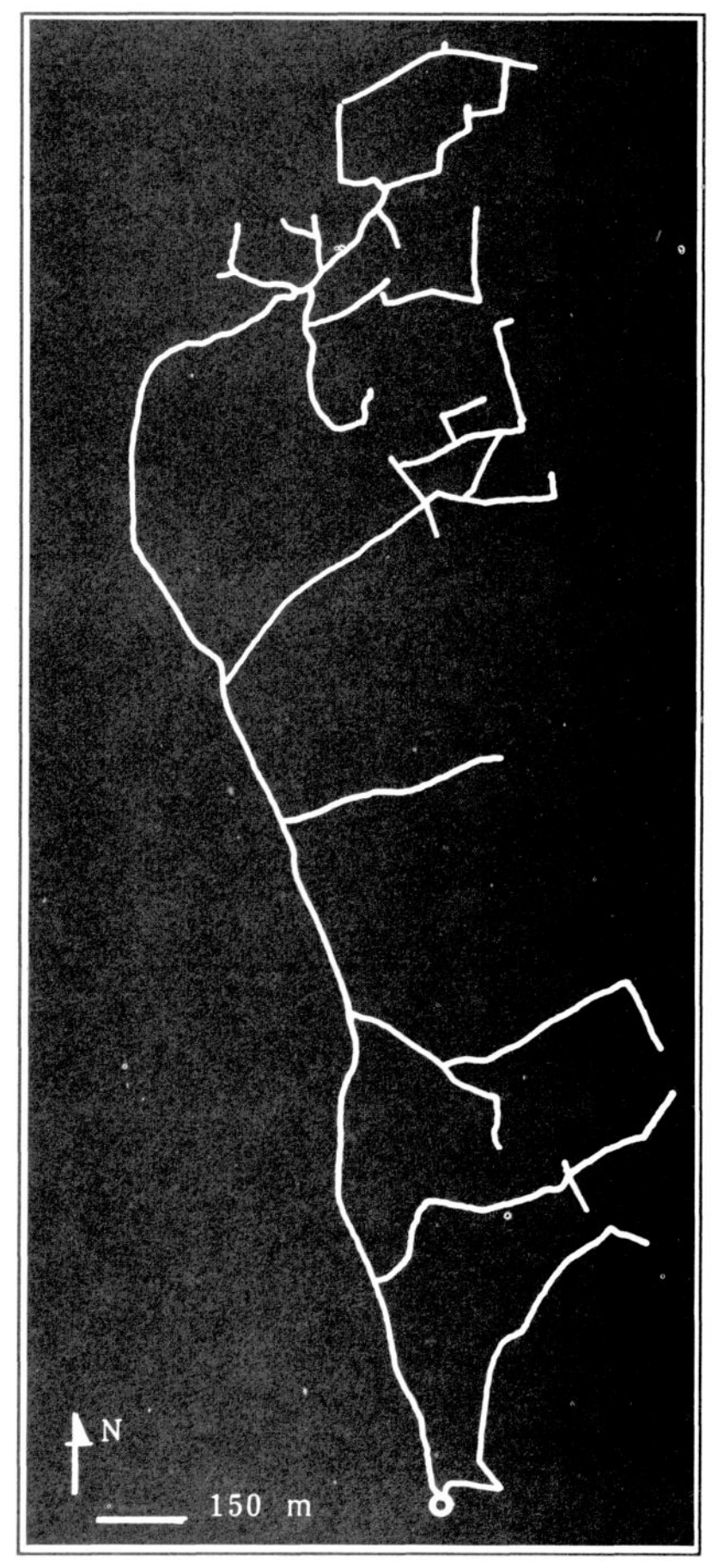

FIGLRE 7: IDARIDIIIY, commune situće au Nord-Ouest de Lyon $D=1.17, e-2 \%$ 1981 .

2. Serge Thibault, Modélisation morpbo-fonctionnelle des réseaux d'assainissement urbain à l'aide du concept de dimension fractale. Thèse D.E., Institut National des Sciences appliquées de Lyon. Université de I.yon I, 1987, 305pp. 
Thibault - Fractals

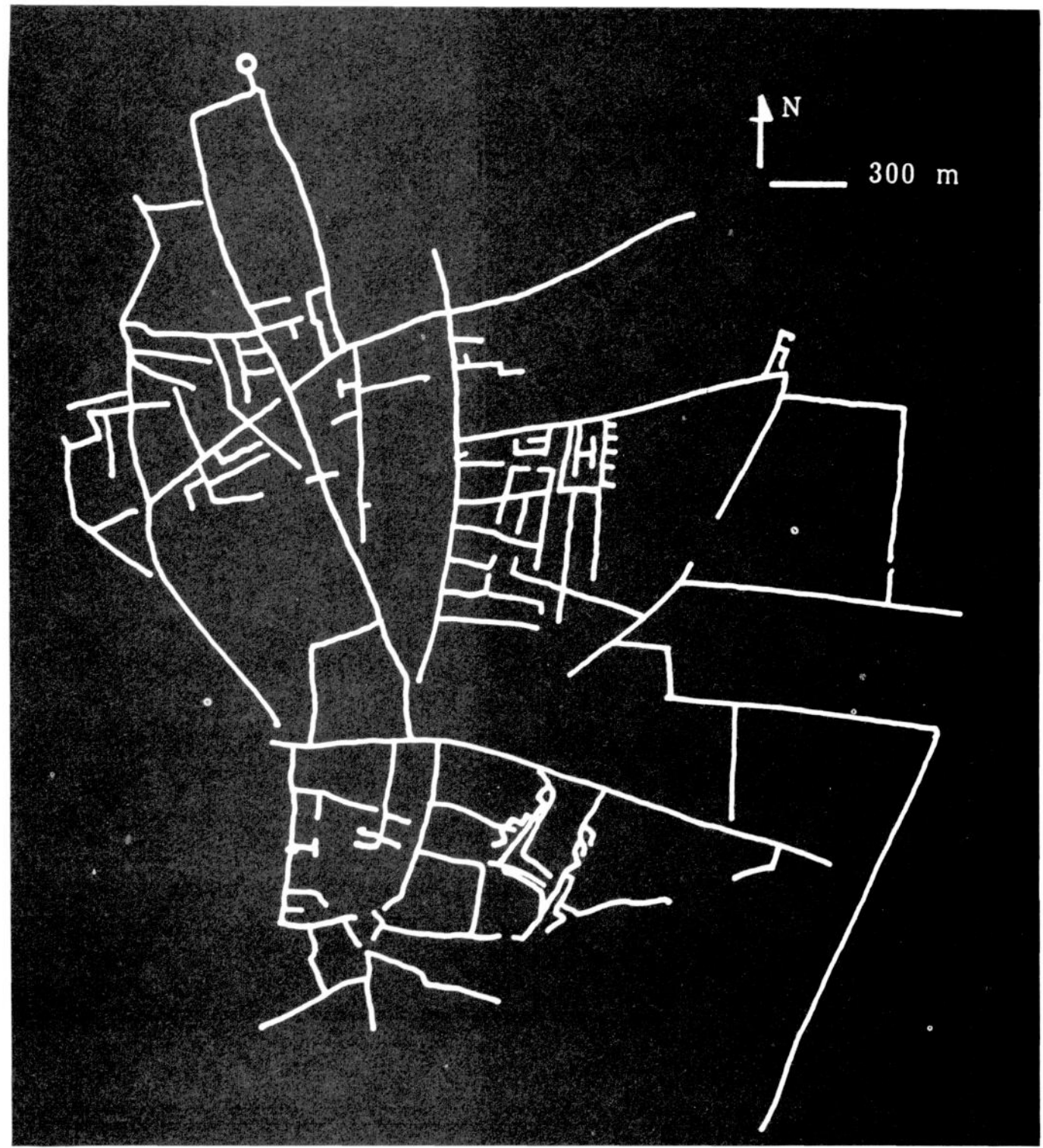

FIGURE 8: MEYZIIEU, commune de la plaine de l'Est de Lyon $\mathrm{D}=1.86, \mathrm{e}-7 \%$

3. Michel Desbordes, Réflexions sur les métbodes de calcul des réseaur urbains d'assainissement pluvial. Thèse D.I., Université des Sciences et Techniques du Languedoc. UER IX, Montpellier, 1976, 203pp.

\section{Thibault, 1987.}

5. J.S. Smart, "Channel Networks," Advances in Hydroscience (1972), Vol. 8, pp. 305-346.

6. Benoît Mandelbrot, Les objets fractals, 2ème éd. (Paris: Flammarion, 1984)

7. Gartner Martin, Le monde mathématique (Paris: Ed. Belin), pp. 98-100.

8. Mandielbrot.

9. Thibault.

10. M. Dubois, P. Atten, P. Berge, "Lordre chaotique", La Recherche, Vol. 18, ํㅜ 185 (1987), pp. 198-201.

11. Gabricl Dupuy, Systèmes, réseaux et territoines (Paris: Presses de l'ENPC, 1985). 
FLUX 4 Avril-Juin 1991

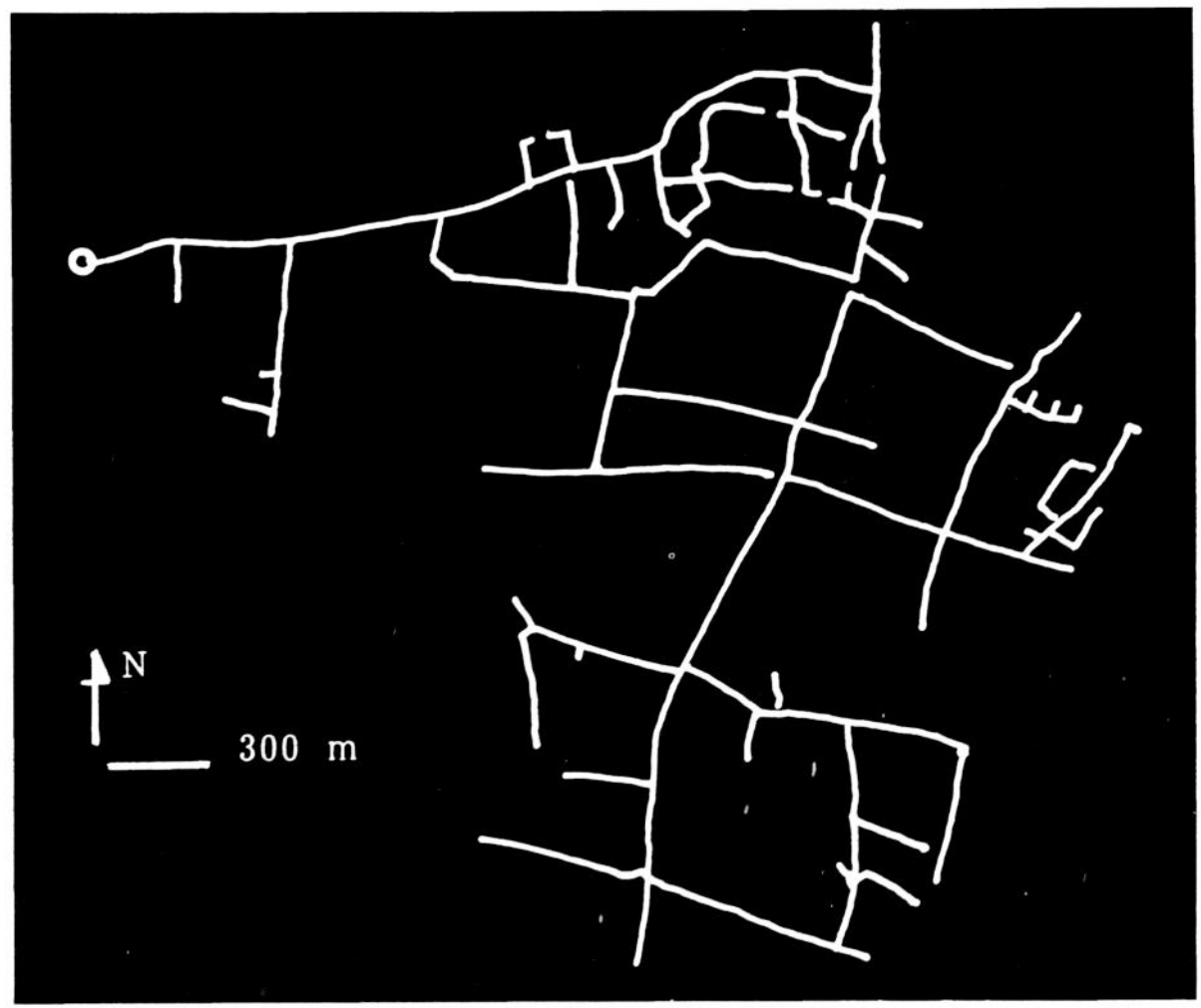

FIGURE 9: CHASSIEU, commune de la plaine de l'Est de Lyon $D=1.68, \mathrm{e}-5 \%$

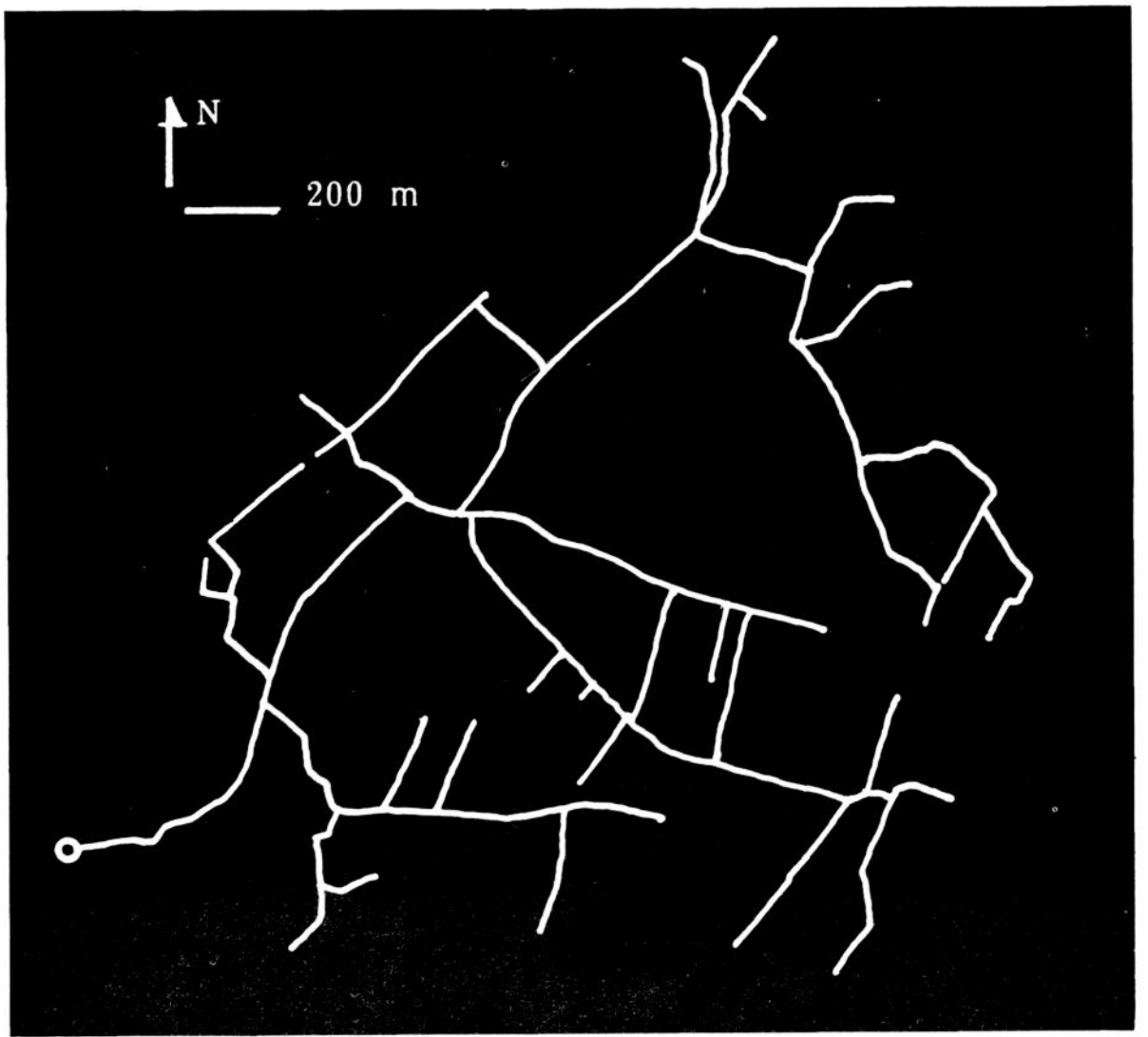

FIGURE 10: SAINTE-FOY-LES-LYON, commune situće au Sud-Ouest de Lyon $\mathrm{D}=1.49, \mathrm{e} \sim 2 \%$ 